INPLASY

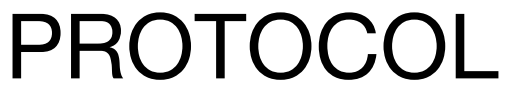

To cite: Zeng et al. Clinical Evidence of Acupuncture and Related Therapy in Patients with Cancer-pain: A Protocol for Systematic Review and Meta-analysis. Inplasy protocol 202090073. doi:

10.37766/inplasy2020.9.0073

Received: 20 September 2020

Published: 20 September 2020

Corresponding author:

Guohua Lin

tcmlin-801@163.com

Author Affiliation:

Department of Acupuncture,

The First Affiliated Hospital,

Guangzhou University of

Chinese Medicine,

Guangzhou, China

Support: None.

Review Stage at time of this submission: Preliminary searches.

Conflicts of interest:

No conflict of interest.

\section{Clinical Evidence of Acupuncture and Related Therapy in Patients with Cancer-pain: A Protocol for Systematic Review and Meta-analysis}

Zeng, J1; Zhou, R²; Luo, Z³; Zhang, N4; Liu, Z5; Lin, G6.

Review question / Objective: To investigate whether acupuncture and related therapies are effective and safe in relieving pain in patients with cancer pain, improving their quality of life, psychological problems (such as anxiety and depression), and reducing the dosage of analgesics.

Condition being studied: There are 7 million new cases of cancer every year worldwide. Most cancer patients suffer from moderate to severe pain. Nearly $70 \%$ of patients with cancer pain receive no effective analgesic treatment.In China, about 1 million people suffer from cancer pain each year, and $60 \%$ to $90 \%$ of patients go to the hospital for it. These patients with cancer pain also present insomnia, anxiety, depression and other psychiatric symptoms. Cancer pain not only diminishes patients' quality of life and physical function, but also incurs economic costs to both their families and to society.

INPLASY registration number: This protocol was registered with the International Platform of Registered Systematic Review and Meta-Analysis Protocols (INPLASY) on 20 September 2020 and was last updated on 20 September 2020 (registration number INPLASY202090073).

\section{INTRODUCTION}

Review question / Objective: To investigate whether acupuncture and related therapies are effective and safe in relieving pain in patients with cancer pain, improving their quality of life, psychological problems (such as anxiety and depression), and reducing the dosage of analgesics.

Condition being studied: There are 7 million new cases of cancer every year worldwide. Most cancer patients suffer from moderate to severe pain. Nearly $70 \%$ of patients with 
cancer pain receive no effective analgesic treatment.In China, about 1 million people suffer from cancer pain each year, and $60 \%$ to $90 \%$ of patients go to the hospital for it. These patients with cancer pain also present insomnia, anxiety, depression and other psychiatric symptoms. Cancer pain not only diminishes patients' quality of life and physical function, but also incurs economic costs to both their families and to society

\section{METHODS}

Participant or population: Adult patients (age $=18$ years old) with either sex who diagnosed with cancer. Pain could be reported at their entrance of the study or not. The pain could be induced by the cancer itself such as progression of tumor; or it could be induced by the treatments of cancer, for instance, chemotherapy or surgery.

Intervention: Acupuncture and related therapies are defined as acupuncture techniques and meridian acupoint stimulation methods, including acupuncture, electroacupuncture, ear acupuncture, five element needle, wristankle acupuncture, fire needle, moxibustion, acupoint acupressure, transcutaneous electrical acupoint stimulation (TEAS), tui-na, massage and combined interventions.

Comparator: Blank control, placebo control, sham acupuncture control, and analgesics control are the comparator interventions. The placebo will be a needle attached to the surface of the skin that does not penetrate the skin.27 Sham acupuncture is defined as placing the needle in an area near, but not at, the acupuncture point, or the electrical stimulation of the skin above local acupoints by electrodes attached to the skin. Simple local skin touching. We will investigate the following comparisons: a.The experimental group and the control group each had routine nursing, and the effect was evaluated either with or without acupuncture intervention. b.Acupuncture and related therapies are only compared to placebo or sham treatment. c.Acupuncture and related therapies plus active treatment or drug treatment, compared with active treatment or drug treatme t. d.Acupuncture and related therapies plus active treatment methods or drugs, compared with placebo or sham treatment plus active treatment methods or drugs.

Study designs to be included: Randomized controlled trials will be included.

Eligibility criteria: We will conduct our meta-analysis in accordance with the Cochrane Handbook for Systematic Reviews of Interventions and report this meta-analysis based on the PRISMA (Preferred Reporting Items for Systematic Reviews and Meta-analyses) guidelines.

Information sources: Electronic searches Published RCTs will be retrieved by searching the Central Registers of Medline, Embase, and Cochrane Controlled Trials. We will use the following search terms: randomized controlled trial, cancer pain, acupuncture, electroacupuncture, ear acupuncture, five element needle, wristankle acupuncture, fire needle, moxibustion, acupoint acupressure, transcutaneous electrical acupoint stimulation (TEAS), tui-na, and massage. Languages will be restricted to English. See Table 1 for the search strategy. Other sources Similar meta-analyses have been published, with references to neuropathic pain textbooks, alternative and complementary medicine textbooks, and clinical guidelines for related trials set by the WHO's International Clinical Trials Registry Platform (ICTRP).

Main outcome(s): Pain relief as a curative effect, defined by original studies. The change in grade and dosage of analgesics for cancer pain. Adverse events.

Quality assessment / Risk of bias analysis: The risk of bias in six areas (sequence generation, allocation hiding, blindness, incomplete data evaluation, selective results reporting, and other sources of bias) will be assessed with the Cochrane Deviation Risk Collaborative Tool. This will 
provide reasons to judge potential risk. Any disagreement will be resolved by discussion with a methodology expert.

Strategy of data synthesis: Studies will be screened by two independent researches who are familiar with the literature management tools. The two authors' measurement consistency in making simple inclusion/exclusion decisions will be calculated with Kappa Statistics. Kappa values between $0.40-0.59$ are considered consistent, between $\mathbf{0 . 6 0 - 0 . 7 4}$ are fairly good, and 0.75 and above are very good. The two authors will review and screen the titles and abstracts to identify eligible trials. According to the inclusion criteria, duplicates will be removed with EndNote (v.x9.0). If necessary, the full text will be read, the exclusion studies will be listed in a table, and the reasons for the exclusion, and any differences, will be discussed with a methodology expert.

Subgroup analysis: Subgroup analysis will be based on intervention (acupuncture, electroacupuncture, ear acupuncture, five element needle, wrist-ankle acupuncture, fire needle, moxibustion, acupoint acupressure, transcutaneous electrical acupoint stimulation (TEAS), tui-na, massage) or overall bias. The severity of the cancer pain will also be considered.

Sensibility analysis: We will conduct a sensitivity analysis to verify the robustness of the research conclusions, assess the methodological quality, the study design, the effect of sample size and missing data, and the effect of the analysis method on the results of this review. The metaanalysis will be repeated, and lower quality studies will be excluded. These results will then be compared and discussed.

Language: There is an English language summary.

Country(ies) involved: China.

Keywords: Acunpuncture; Cancer-pain.
Author 1 - Jingchun Zeng - drafts the manuscript.

Author 2 - Runjin Zhou - provides statistical expertise.

Author 3 - Zhenke Luo - establishes search strategy.

Author 4 - Na Zhang - contributes to the development of selection criteria and the risk of bias assessment strategies.

Author 5 - Zijun Liu - contributes to the development of selection criteria and the risk of bias assessment strategies.

Author 6 - Guohua Lin - contributes to the development of selection criteria and the risk of bias assessment strategies.

Contributions of each author: 